Article

\title{
The Cybersemiotics and Info-Computationalist Research Programmes as Platforms for Knowledge Production in Organisms and Machines
}

\section{Gordana Dodig Crnkovic}

Mälardalen University, School of Innovation, Design and Engineering, Sweden;

E-Mail: gordana.dodig-crnkovic@mdh.se

Received: 23 February 2010; in revised form: 1 April 2010 / Accepted: 12 April 2010 /

Published: 13 April 2010

\begin{abstract}
Both Cybersemiotics and Info-computationalist research programmes represent attempts to unify understanding of information, knowledge and communication. The first one takes into account phenomenological aspects of signification which are insisting on the human experience "from within". The second adopts solely the view "from the outside" based on scientific practice, with an observing agent generating inter-subjective knowledge in a research community. The process of knowledge production, embodied into networks of cognizing agents interacting with the environment and developing through evolution is studied on different levels of abstraction in both frames of reference. In order to develop scientifically tractable models of evolution of intelligence in informational structures from pre-biotic/chemical to living networked intelligent organisms, including the implementation of those models in artificial agents, a basic level language of Info-Computationalism has shown to be suitable. There are however contexts in which we deal with complex informational structures essentially dependent on human first person knowledge where high level language such as Cybersemiotics is the appropriate tool for conceptualization and communication. Two research projects are presented in order to exemplify the interplay of info-computational and higher-order approaches: The Blue Brain Project where the brain is modeled as info-computational system, a simulation in silico of a biological brain function, and Biosemiotics research on genes, information, and semiosis in which the process of semiosis is understood in info-computational terms. The article analyzes differences and convergences of Cybersemiotics and Info-computationalist approaches which by placing focus on distinct levels of organization, help elucidate processes of knowledge production in intelligent agents.
\end{abstract}


Keywords: knowledge generation; cybersemiotics; info-computationalist naturalism; information modeling; observation; measurement; simulation; reality as interface

PACS Codes: $01.70 .+$ w Philosophy of science, 05.45.Gg Control of chaos, applications of chaos, 05.65.+b Self-organized systems (see also 45.70.-n in classical mechanics of discrete systems), 07.05.Fb Design of experiments, 07.05.Tp Computer modeling and simulation, S 01.75.+m Science and society, 87.10.Vg Biological information

\section{Cybersemiotics and Info-Computationalism}

(W)e must do more than include physics, chemistry, and biology with their functional explanations into our definition of science: we need to embrace a Peircian biosemiotic interpretation of biology. Cybersemiotics combines this with a theory of levels, information, semiotics, and language interactions. This means that we do not dismiss the results obtained so far, but rather that we relativise their implicit frameworks and integrate the science into a new framework while adding semiotics. Brier [1] p. 431.

In his Cybersemiotics, Brier presents 'a metascientific framework which is also meta to the humanities and the social sciences' ([1] p. 7) and which forms a unified conceptual framework for meaning, cognition and communication. The Cybersemiotics programme proposes a dialogue between different knowledge systems in which science has its given place, complemented by other knowledge systems deriving their meaningfulness from premises found at different levels of abstraction. This reconciliatory attitude is in concord with the idea of hypercomplexity of Qvortrup [2]. According to Qvortrup there are four different orders of knowledge: the first order knowledge (knowledge about something, object level knowledge); the second order knowledge (knowledge about the conditions of knowing, methodological knowledge); the third order knowledge (knowledge about the conditions of the reflective knowledge, creative knowledge), and the fourth order knowledge (the world knowledge or the knowledge horizon, metaphysics). In the succession of knowledge orders, higher levels of organization must be addressed on the higher level of abstraction where more expressive languages are necessary than those used to describe basic mechanisms.

Stability in society encompassing various sorts of knowledge systems and practices is not achieved by the hegemony of a strong center providing the one possible true knowledge, but by offering the means for meaningful communication by mutual observation. The functional objective of society is not to create "order" out of “chaos," but to manage complexity by complexity” ([2] pp. viii-ix).

In this process of management of complexity by complexity different stakeholders in knowledge-production are evolving, learning from each other, adopting new knowledge and developing new strategies. The boundaries between research fields are becoming increasingly diffuse as different kinds of trans-disciplinary, cross-disciplinary and inter-disciplinary knowledge are developing, bridging CP Snow's gap between cultures. 
In what follows, I will argue that Info-computationalist framework provides a fundamental level of description for knowledge generation capable of capturing its mechanisms [3]. The comparison between Cybersemiotics and Info-computational accounts of knowledge production may also be seen as being about the first-person and third-person accounts, a topic addressed by Chalmers [4] indicating his dissatisfaction by third-person accounts being about "appearances" [5]. Nevertheless, third person accounts are inevitable. We make our accounts as third persons regularly in relation to everything in the world except for ourselves. We also ascribe intelligence and sentience to other people without really knowing the mechanisms of their thoughts and feelings. Finally our first person accounts are not especially trustworthy source of knowledge. In everyday life, the richness and originality of first-person accounts of individuals is balanced by third-person accounts of the rest of the stake holders in knowledge production. In a culture both kind of accounts are contributing.

Knowledge production of the sciences is based on third-person accounts alone. Moreover, in the physical sciences reductionist approaches prevail. Reducing phenomena to fundamental constituent parts and their interactions is the main goal of a reductionist project as it radically delimits the space of possible models Anderson [6]. Interestingly enough, all the success notwithstanding, classical physical sciences have not yet succeeded in explaining life processes and the behaviors of systems of living beings and their interactions such as they appear in e.g., biology, ecology, psychology, economy or social sciences. The key problem is tackling complexity of living processes and their interactions compared to simplicity of natural symmetries found on the fundamental physical level [7]. The hope is that we will be able to gain substantial insight into the nature of complex systems by an Info-Computationalist approach [8,9]. Unlike the classical mechanistic framework in physical sciences, Info-Computationalism relies on more complex mechanisms capable of accommodating self-reflective processes such as auto-poesies and is thus suitable for modeling living systems and also for unified understanding of natural structures and processes. A detailed account of Info-Computationalism as a framework capable of tackling living systems including phenomena of self-organization and other self-reflective processes is given in [9], and it also answers Hofkirschner [70]. It starts from ideas of cybernetics and generalizes information and computation to become two basic complementary ontological principles. Info-Computationalism presents a synthesis of Floridi's Informational Structural Realism [10,11] and (Pan)Computationalism of Chaitin, Wolfram and Lloyd [12-14].

Brier correctly characterizes the classical cybernetics informational approach in the following way:

The cybernetic computational informative view is based on universal and abstract (un-embodied) conceptions of information and computation that is the foundation of "the information processing paradigm”, which is foundational for much cognitive science and its latest developments into brain function and linguistics comprising also a philosophy of science. It is claiming that "information" is the objective forms of the world. That it can be objectively parted into pieces of "data" and that humans, brains, computers and organizations process them in the basic same way, which is called "computation" and is a basic - not really well-defined concept_of information processing that goes beyond the Turing computer definition. Anyway it is a "software" definition opposed to a "hardware" definition (metaphors again). Brier [15]. 
However, for the Info-Computationalism of Dodig Crnkovic [8] and Dodig Crnkovic and Müller [9] computation is not any longer just an abstract symbol manipulation, but any information processing occurring on the informational structure of the universe. It is based on Informational Structural Realism (ISR) of Floridi [10] in the following way:

A preferable alternative is provided by an informational approach to structural realism, according to which knowledge of the world is knowledge of its structures. The most reasonable ontological commitment turns out to be in favour of an interpretation of reality as the totality of structures dynamically interacting with each other. Floridi [11] p. 151.

Also very important from the point of view of information as a difference:

The relation of difference seems a precondition for any other relation and hence for any process of knowledge. Relata as merely differentiated entities and nothing else (at least not yet) are possible only because there is a relation of initial, ontological difference in the first place. Floridi [10] p. 234.

A significant consequence of ISR is that, as far as we can tell, the ultimate nature of reality is informational, that is, it makes sense to adopt Levels of Abstraction that commit our theories to a view of reality as mind-independent and constituted by structural objects that are neither substantial nor material (they might well be, but we have no need to suppose them to be so) but cohering clusters of data (not in the alphanumeric sense of the word, but in an equally common sense of differences de re, i.e., mind-independent, concrete, relational points of lack of uniformity). Structural objects work epistemologically like constraining affordances: they allow or invite certain constructs (they are affordances for the information systems) [ibid 154].

What Floridi assumes to be mind-independent data corresponds to proto-information of Info-Computationalism. Reality is informational and mind-independent and consists of structural objects, in the sense of ISR which unifies metaphysical views of Wiener ("information is information, not matter or energy") and Wheeler (“it from bit”).

Computation, on the other hand is the dynamics of informational structure. Here is how Toffoli describes computation:

Coming back to the main discussion, we may observe that computation seems to be everywhere but what is it precisely? A common way to tell a computation is by what Aristotle would have called its 'efficient cause' what today we'd call its proximate mechanisms. Tofoli [16]. See also [17].

Computation is present in the entire universe, according to Chaitin:

And how about the entire universe, can it be considered to be a computer? Yes, it certainly can, it is constantly computing its future state from its current state, it's constantly computing its own time-evolution! And as I believe Tom Toffoli pointed out, actual computers like your PC just hitch a ride on this universal computation! Chaitin [12]. See [17]. 
Within Info-Computationalism, computation is both symbolic and sub-symbolic information processing, such as Natural Computation or Organic Computation. Symbolic information processing is characterized by rules operating on discrete, static tokens, while sub-symbolic information processing applies to learned, fuzzy rules on continuous, distributed representations. Sub-symbolic paradigm is especially important in Biologically Inspired Computing. In sub-symbolic (connectionist) theory, information is processed in parallel by simple computations performed by e.g., neurons in a neural network. Information is represented by a sequence of pulses, e.g., pixel images or other data streams of sensor inputs which consists a basis for behavior control [20]; Hofstadter's term is subcognition.) As computation in general corresponds to the dynamic of the processes that exist in the universe, it is necessarily both discrete and continuous. In the Info-Computational naturalist framework, information and computation are two fundamental and inseparable elements necessary for naturalizing meaning, cognition and mind; see Dodig Crnkovic [19]. Information is as already argued the structure, the fabric of reality. The world exists independently from us (realist position of structural realism) in the form of proto-information or ur-(in)formation, the richest potential form of existence corresponding to Kant's Ding an sich. That proto-information becomes information (which, according to Bateson is a difference that makes a difference, see [21]) for a cognizing agent in a process of interaction through which specific aspects of the world get uncovered.

In science this process of successive transformation of manifold potential proto-information in the world into particular actual information in an agent becomes evident when for example observing the same physical object in different wavelengths [21]. The world as it appears to an agent is dependent on the type of interaction through which the agent acquires information. The potential information in the world is obviously much richer than what we observe, containing invisible worlds of molecules, atoms and sub-atomic phenomena, distant cosmological objects and similar. Our knowledge about this proto-information which is revealed with help of scientific instruments will surely continue to increase with the development of new devices, i.e., the new ways of interaction with the world [19].

Computation and information are the backbone of the sciences. They are inseparable - there can be no structure without a process (a structure actualizes or gets uncovered through a process) and the vice versa, there is no process without a structure to act on [8,22]. Formalization of Info-computational approach within Category Theory is done by Burgin and may be found in [23].

In what follows the present state of the theory of Info-computationalist naturalism will be presented taking into account self-reflective, self-referential knowledge production mechanisms and the role of first person contra third person observers.

\section{Info-Computational Naturalism and Knowledge Generation}

Within the Info-computational framework knowledge is seen as a result of successive structuring of input data, where data is understood as the most basic information units, signals acquired by a cognizing agent through the senses/sensors/instruments. Information is meaningful data, which in the next step of organization can be turned into knowledge by an interactive computational process occurring in the agent. (The view of information as meaningful data comes from engineering and can be questioned in this more general context. As already described, for an agent, the reality presents proto-information or the potential information which agent interacts with and is indeed part of. A lot of 
information processing takes place on the sub-symbolic level and one may ask what sort of meaning could be ascribed to sub-symbolic information. Also what about meaningless data? On a basic level meaningless data do not present any information. However there are always meta levels on which meaning can be found such as e.g.,: this apparatus must be broken as it gives totally meaningless data.) So, potential information exists in the world even if no cognizing agents are present. As already mentioned, the world (reality) for an agent is potential information. Knowledge on the other hand always resides in a cognitive agent. An agent is a physical system, living organism or a robot possessing cognitive structures capable of adaptive behaviors. Living agents evolved successively starting with prebiotic evolution of increasingly complex molecules able to adapt to the environment and reproduce. Semantics of information is relative to an agent.

The meaning of proto-information for a biological organism is the use the agent (organism) has for it. Information is always embodied in a physical signal, molecule or event which will induce change of structure or behavior of an agent. Information about the sugar gradient in the environment has high relevance and is meaningful for an organism which uses sugar as a source of energy. Semantics develops as a part of data (signals from the physical environment and from their own physical body)/information/knowledge structuring process, in which complex structures are self-organized by the computational processing of simpler ones.

The meaning of information is thus defined for an agent and a group of agents in a network (ecology) and it is defined by the role information plays for them. Knowledge generation in biological agents presupposes natural computation, defined by MacLennan [24] as computation occurring in nature or inspired by that in nature, which is the most general current computing paradigm.

Taking information and computation as basic principles in a dual-aspect Info-computational model, a common framework is formulated in [8] and [19] which provide arguments that knowledge generation should be understood as natural computation. Knowledge is a characteristic of intelligent agents; intelligence in living organisms being a matter of degree. Artificial intelligent agents can be ascribed knowledge as long as they function as agents, exchanging information with the world. In the same way as we do not ascribe knowledge to a dead living organism, we do not ascribe knowledge to a non-functional robot. Information, on the other hand can be stored in non-living systems, but for information to be functional knowledge, a process of access and interaction between different pieces of information is necessary.

It is important to notice that when we talk about computation in living organisms, models of computation beyond Turing machine are needed to handle complexity of phenomena such as metabolic processes, perception, learning, intelligence, consciousness, social phenomena, etc. The source of inspiration for new models of computing is found in nature and especially in organic processes understood as computing. Evolutionary development of increasingly intelligent self-organized structures and processes has enabled living organisms to survive in the complex and dynamical world.

All of our knowledge of the world is based on information we gain from the world, be it from personal experience or learning (by getting information) from other people. Physicist Zeilinger suggest the possibility of seeing information and reality as one:

In the history of physics, we have learned that there are distinctions that we really should not make, such as between space and time... It could very well be that the distinction we 
make between information and reality is wrong. This is not saying that everything is just information. But it is saying that we need a new concept that encompasses or includes both [25].

Consequently, we should not make a distinction between reality and information, even though Zeilinger leaves open the possibility that reality is more than information. This is in accord with Floridi's Informational Structural Realism which says that reality is made of informational structures [10] and [11] as well as with the Info-computational ontology, based on Informational Structural Realism with information as the fabric of reality.

\section{Information and Computation in Biological and Intelligent Artificial Systems}

If we take a dead bird and throw it up into the air its path describes parabola, in conformity with the laws of motion (...). Take a living bird and throw it up in the air and something entirely different happens. Fundamentally, it is matter of understanding how, given a physical universe dominated by matter and energy, systems can emerge that determine their own behavior by means of information or computation. Emmeche [26].

Recent studies in biology, ethology and neuroscience have increased our knowledge of biological cognitive functions and led to the conclusion that the most important feature of cognition is its ability to deal efficiently with complexity, according to Gell-Mann [27]. Insights into natural intelligence, together with the dramatic increase in power of electronic computing bring us closer to the modeling and simulation of intelligent behavior which is the goal of Artificial Intelligence (AI) based on the belief that intelligent behavior can be understood in such a way that a machine can be constructed able to simulate it [22]. Goertzel [28] argues from the computationalist point of view that intelligence may be seen as based on several levels of data processing in a cognizing agent, animal or a machine/AI agent. Information (sensory data processed by an agent) can be understood as an interface between the data (world) and an agent's perception of the world [29].

It is argued that Info-Computationalism is the most appropriate theoretical framework for understanding of intelligence [22]. Patterns of information are attributed both to the world and to the functions and structures of the brain. Knowledge can be seen as an interface between perception and cognition. Structures of knowledge are related both to percepts (sensory information) and to the cognitive functions and organization of the brain. Meaning and interpretation are the results of the processes of temporal changes of information, its structural organization and establishment of relations to already existing information. In other words, the meaning of an object in the external world is realized through the perception of sensory signals, their processing through nervous system, comparison with memorized objects, anticipation from memorized experiences of possible scenarios in which the object played different roles, etc.

According to Minsky [30] data, information, perceptual images and knowledge are organized in a multiresolutional (multigranular, multiscale) model of the brain and nervous system. Multiresolutional representation has proven to be a good way of dealing with complexity in biological systems, and they are also being implemented in AI (Goertzel).

Our understanding of cognition and above mentioned processes can be tested in a rigorous manner in Cognitive Robotics research. From Cognitive Robotics it is becoming evident that intelligence is 
closely related to agency. Anticipation, planning and control are essential features of intelligent agency. A similarity has been found between the generation of behavior in living organisms and the formation of control sequences in artificial systems.

Self-organization, self-reproduction and self-description (or self-representation) are vital intrinsic properties of natural intelligent systems. More and more of intelligent robotics builds on similar basic principles called organic computing, which answers in positive the question Hofkirchner puts forward in [70] “Does Computing Embrace Self-Organisation?”. Learning is an essential part of each of the above three self-x capabilities and it requires on the higher level of organization the development of a symbolic system which is easy to maintain and use. It is possible to build intelligent control systems that can collect and process information, as well as generate and control behavior in real time, and cope with situations that evolve among the complexities of the real world. Generation of structures resulting from sensory processes (data), and information organized into knowledge to be used in decision making are built in a multiresolutional way, with many pattern recognition and control mechanisms hardwired. In intelligent biological systems based upon a hierarchy of functional loops, each of these loops can be treated as a control system per se, [30]. Goertzel [28] hypothesizes that (intelligent) mind is basically a superposition of two systems: a structurally associative memory and a multilevel perceptual-motor control process hierarchy. These two correspond to informational structure and computational process. By superposing two systems, according to Goertzel, mind emerges combining memory (structure) and a process (dynamic control).

Along with the above engineering approaches to AI with roots in logic and programming, there is one which tries to implement in silico info-computational biological functions by computationally modeling biological structures starting from molecular level up to neurons and neo-cortical columns notably the Blue Brain Project of ETF, discussed later on.

Up to now only specific aspects of intelligence (weak AI) have been developed, while general AI seems still a far-reaching goal, after more than fifty years of research.

\section{Knowledge Generation as Natural Computation}

Classical theoretical model of a computer is represented by a Turing machine which is equivalent to an algorithm/effective procedure, recursive function or formal language. A Turing Machine is a logical device for execution of algorithms. (A "Logical device” is Turing's own name for his "machine” i.e. model of computation.) On the other hand biological structures and processes understood as embodied physical information processing are physical, highly interactive and networked, both discrete and continuum and thus in general do not resemble Turing Machines in the first place. In order to develop a general theory of the networked physical information processing, we must also generalize the ideas of what computation is and what it might be. For new computing paradigms, see Wegner [31], Kurzweil [32] and Dodig-Crnkovic [19]. Compared with new computing paradigms, Turing machines form the proper subset of the set of information processing devices.

Present day's computers perform syntactic mechanical symbol manipulation which in the future has to be extended to include information processing with semantic aspects. Burgin, [33] identifies three distinct components of information processing systems: hardware (physical devices), software (programs that govern its functioning), and infoware which represents information processed by the 
system. Infoware is a shell built around the software-hardware core which is the traditional domain of automata and algorithm theory. The Semantic Web is an example of infoware. Bio-computing and especially organic computing are expected to substantially contribute to the infoware by adopting strategies for the handling of complexity found in the organic world.

\section{Info-computational Framework for Evolution of Embodied Knowledge}

But this is just to say that any conception of animal behavior which makes sense of it all will have to see the animal's cognitive equipment as serving the goal of picking up and processing information. And this commits one to the notion of animal knowledge. Kornblith [34].

Naturalized epistemology according to Harms [35,36] is an idea that knowledge should be studied as a natural phenomenon. The subject matter is not our concept of knowledge, but the knowledge itself as it appears in the world. An evolving population incorporates information about its environment through changes by natural selection, as the state of the population actually matches the state of the environment. The variants which adapt and survive in the environment increase their frequency in the population. Harms [35] proves a theorem showing that natural selection will always lead a population to accumulate information, and so to 'learn' about its environment. Okasha points out that

any evolving population 'learns' about its environment, in Harms' sense, even if the population is composed of organisms that lack minds entirely, hence lack the ability to have representations of the external world at all [37].

That is correct, and may be understood not as a drawback but as strength in virtue of generality of a naturalistic approach. It shows how mind is a matter of degree and how it slowly and successively develops with evolution. There is fresh evidence that even simple 'lifeless' prion molecules are capable of evolutionary change and adaptation [38].

However, as both Harms and Okasha agree, this understanding of basic evolutionary mechanisms of accumulating information and at the same time increasing information processing capacities of organisms (such as memory, anticipation, computational efficiency) is only the first step towards a full-fledged evolutionary epistemology, but it is a most difficult and significant first step. From bio-computing we learn that in living organisms biological structure (hardware) is at the same time a program (software) which controls the behavior of that hardware.

By autopoetic process, biological system changes its structures and thus the information processing patterns in a self-reflective, recursive manner. Maturana and Varela give the following description:

An autopoietic machine is a machine organized (defined as a unity) as a network of processes of production (transformation and destruction) of components which: (i) through their interactions and transformations continuously regenerate and realize the network of processes (relations) that produced them; and (ii) constitute it (the machine) as a concrete unity in space in which they (the components) exist by specifying the topological domain of its realization as such a network [46]. 
Natural selection of organisms, responsible for nearly all information that living systems have built up in their genotypes and phenotypes, is a simple but costly method to develop knowledge capacities. Higher organisms (which are "more expensive" to evolve) have developed learning capability as a more efficient way to accumulate knowledge. The difference between "genetic learning" (typical of more primitive life forms) and acquisition of cognitive skills on higher levels of organization of the informational structures of the nervous system (such as found in vertebrata) will be the next step to explore in the project of naturalized epistemology.

\section{Interaction of an Agent with the Environment and Info-computational Evolution}

How does information acquire meaning naturally in the process of interaction of an organism with its environment? As argued in Bates [39] mechanisms of naturalized knowledge generation are found via study of evolution and related with cognitive, linguistic and social structures of living beings, from the simplest ones to those at the highest levels of organization. An agent (an organism or an artificial one) receives inputs (data) from the physical environment and interprets these in terms of its own earlier experiences, comparing them with stored data in a feedback loop. Through that interaction between the environmental data and the inner structure of an agent, a dynamical state is obtained in which the agent has established the representation of the current state. The next step in the loop is to compare the present state with one's own goals and preferences (saved in memory). This process is related with the anticipation of possible actions and their consequences. Normally this takes time, but there are obvious exceptions. Reactions to situations where an agent is in danger are usually hard-coded and connected via a short-cut activating an immediate, automatic, unconscious response.

For a living organism, the efficiency of the computational process is decisive for survival. Studies of vision show economy of cognition which focuses on that part of the scene which is variable and dynamic, and uses memorized data for the rest which is static (here again only a difference is observed that makes a difference). Our cognition is an emergent phenomenon resulting from both memorized and observed data. Considering chunks of structured data as building blocks, instead of performing lengthy computations on those data sets in real time gives a powerful speed-up. With each higher level of organization, the computing competency of an organism's cognitive apparatus gets further increased. The efficiency of meta-level structures and processes is becoming explicitly evident in computational modeling.

Cognition is characteristics of all living systems which organize themselves by interacting with their environment (enactivism of Maturana and Varela): Knowing is being is doing (acting/agency). The following two quotes illustrate this position:

This circularity, this connection between action and experience, this inseparability between a particular way of being and how the world appears to us, tells us that every act of knowing brings forth a world ... all doing is knowing, and all knowing is doing. Maturana and Varela [47], p.26.

Every interaction of an organism, every behavior observed, can be assessed by an observer as a cognitive act. In the same way, the fact of living-of conserving structural coupling 
uninterruptedly as a living being - is to know in the realm of existence. In a nutshell: to live is to know (living is effective action in existence as a living being).” [47] p. 174.

The "knowledge" of an organism about its world depends not only on the world, but also on the actions the organism is capable of. Interactivism as defined by Bickhard and Kulakov and Stojanov $[40,41]$ is an approach especially suited to the analysis of agency, and in many respects close to the ides of enactivism. It involves naturalism, which means that the physical world (matter) and mind are integrated by interaction. The interactive model offers a critique of correspondence or "spectator" models of cognition, with focus on the consequences of interaction and the emphasis on the process (dynamic) nature of perception (data) as well as information (representation). It is thus related to process metaphysics of Whitehead [42].

The Info-Computationalist approach to naturalized knowledge is based on the fundamental insight of enactivism that cognition in all living beings happens through constant interaction with their environment. This interaction is a process of natural computation. Biological organisms are open complex systems in a regime on the edge of chaos, which is characterized by maximal informational capacity [43]. The characteristic feature of cognizing living organisms is their ability to manage complexity, and to handle complicated environmental conditions with a variety of responses which are results of adaptation, variation, selection, learning, and/or reasoning [27].

As a result of evolution, (starting with molecular evolution), increasingly complex living organisms developed that are able to survive and adapt to their environment. It means that they are able to register inputs (data) from the environment, to structure those into information, and in more complex organisms into knowledge. The evolutionary advantage of using structured, component-based information-processing approaches is improving response-time and efficiency of cognitive processes of an organism. Info-computational naturalized knowledge generation acknowledges the body as our cognitive apparatus understood in terms of structure (information) and processes (computation). All cognition is embodied cognition, in microorganisms as well as in humans [44,45].

Within Info-computationalist naturalism our specific interest is in how the structuring from data to information and knowledge develops in a cognitive agent (a biological organism or an artificial agent) because of its interaction with the environment, including other agents. In accordance with Harms, one can say that living organisms are "about" the environment, as they have developed adaptive strategies to survive and thrive by internalizing environmental constraints. The interaction between an organism and its environment is realized through the exchange of physical signals that might be seen as data, or when structured, as information. Organizing and mutually relating different pieces of information results in knowledge. Info-Computationalism adopts Maturana and Varela's [46,47] theory of autopoetic enactivist cognition as a part of Info-computationalist account.

As all present understanding revolves around cognition and knowledge in the simplest biological organisms the next step in the development of the Info-computationalist model of naturalized knowledge is what Brier rightly points out as missing in present accounts: naturalization of the complex knowledge production in the most complex information-processing agents such as humans augmented by info-computational intelligent machinery. This issue is closely related with our ability to account for human Info-computational cognitive capacities on both individual and group (society, network) level. Moving towards more and more complex cognitive structures and information 
processes, new phenomena emerge which we shall briefly bring up in what follows. Here are some glimpses into the open problems of Info-computational approach to naturalization of human knowledge.

\section{Why Our Perception of the World is an Illusion and What Can We Learn From It?}

Of all information processing going on in our bodies, perception is only a tiny fraction. Our perception of the world depends on the relative slowness of conscious perception. Time longer than one second is needed to synthesize conscious experience. At time scales shorter than one second, the fragmentary nature of perception reveals. The brain creates a picture of reality that we experience as (and mistake for) "the actual thing” [49]. Consciousness provides only a rough sense of what is going on in and around us, focusing in the first place what we take to be essential for us. The world as it appears for our consciousness is a sketchy simulation. Belief that we ever can experience the world directly is the biggest illusion [50].

Hence the brain does not directly map the external world. From this proposition follows the notion of "interpreting brain”, i.e. the notion that the brain must interpret symbols generated by itself even at the lowest level of information processing. It seems that many problems related to information processing and meaning in the brain are rooted in the problems of the mechanisms of symbol generation and meaning. Kaneko and Tsuda [48] p. 192.

What would that mean anyway to experience the world 'directly as it is', without ourselves? Who would experience that? It is important to understand that, as Kaneko and Tsuda [48] emphasize, the brain maps the information about the (part of the) world into itself, but the mapped information is always affected by the activity of the brain itself. The question of what reality is "an sich" in the sense of proto-information "in the wild" and understanding of our interactions with that world outside through the (conscious and sub-conscious) exchange of information is fundamental, and we have to keep searching for a deepened understanding of that reality in relation to ourselves. To that end, awareness of the presence of models (simulations) in our understanding (and perception) of the world is essential.

\section{Observing the Observer Observing Observer and Beyond. Self-reflective Knowledge}

If what we perceive of the world is a simulation our brain plays for us in order to manage complexity and enable us to act efficiently, then our understanding of the world must also be mediated by this modeling nature of cognition. Not even the most reliable knowledge about the physical world as it appears in sciences is independent of the modeling frameworks which indirectly impact what can be known. The common positivist optimism about observations independent of the observer proved problematic in many fields of physics such as quantum mechanics (wave function collapse after interaction), relativity (speed dependent length contraction and time dilatation) and chaos (a minor perturbation sufficient to switch to a different attractor). In general, observer and the systems observed are related and by understanding their relationship we can gain insights into limitations and strengths of models and simulations as knowledge generators, von Foerster [51]. 
Models are simplified representations, made for a purpose and they ignore aspects of the system which are irrelevant to that purpose. The properties of a system itself must be clearly distinguished from the properties of its models. All our knowledge of systems is mediated by models. Engineers using different models often get so familiar with them and their functions that they frequently act as if a model was the actual reality itself [52]. Models have their bounded domains of validity, simplifications and inaccuracies can make them inadequate representations.

Awareness of the modeling character of knowledge and the active role of the cognizing agent in the process of generation of knowledge is of particular interest for Second Order Cybernetics. Cybernetic epistemology is constructivist in recognizing that knowledge cannot be passively transferred from the environment, but must be actively constructed by the cognizing agent based on the elements found in the environment in combination with information stored in the agent. The environment is eliminating inadequate models, which are getting into conflict with the system that uses them. Model construction thus proceeds through variation and selection. This agrees with Ernst von Glasersfeld's two basic principles of constructivism [53].

1. Knowledge is not passively received either through the senses or by way of communication, but is actively built up by the cognizing subject.

2. The function of cognition is adaptive and serves the subject's organization of the experiential world, not the discovery of an 'objective ontological reality'.

According to Glanville [54] the most prominent feature of Second-order Cybernetics (Cybernetics treated cybernetically) is its circularity. The two primary functions that Wiener assigned to Cybernetics, control and communication are fundamentally re-conceptualized. Second Order Cybernetics is proposing a constructivist epistemology as well as ontogenesis (which become constructive through autopoesis).

The key question that arises in this 'constructive' approach lies in the relationship between the virtual world (the model) and reality. The virtual world should not just be an imitation of reality, but a sort of abstraction from reality, and be constructed from our side by utilizing some abstracted essential features of reality. Understanding the relationship between the virtual world and reality is a fundamental issue in the study of complex systems with a constructive approach. Obviously, a virtual world must have some interface with reality [48].

An example from physics can illustrate how observation does not give us the idea of the world "as it is", but provides us with the basis for "reconstruction" we get through the interaction with our environment. When we are observing a very distant star, we are seeing the star as it was in the past, since light travels with finite velocity of about 299,792 km/s and it takes time for photons originating from the star to reach us. It is impossible to see any star as it is "right now". Not even Sun which is eight light minutes away, and even less the next nearest star Proxima Centauri which is 4.3 light-years from the Sun. The mechanism we use for seeing is the detection of light, and light produced at distant places in the universe will reach us after many years of travel through interstellar spaces. As Luhmann rightly noticed: 
There is an external world ... but we have no direct contact with it. [55] p. 64.

This example suggests that the way of observation (such as the distance from the object) influences the results of observation. Even animal perception studies show how the form and function of receptors define what an agent (animal) can perceive. Snakes for example have infra-red sensitivity, so their world looks different from what we see in visible light. All this is important in epistemology, scientific methodology, robotics and number of other fields. [21]

Observer and the system of observation are coupled, as we have already pointed out. The fact is that mutual relationship establishes the result of observation. The next question is: how do we proceed studying the observation itself as a phenomenon? One of the strategies suggested was an introspective approach of an observer, thus observing not only the phenomenon under investigation but even observing herself observing the phenomenon. That is where circularity comes in. Another possible strategy is having an outer (meta-) observer observing the (object-level) observer, and so division of labor typical of classical scientific method. This type of approach can be found in Research of Research (RoR). As science is a self-organized adaptive intelligent learning system, it also incorporates knowledge from meta-level (observers who learn about observation as a phenomenon) to be used by researchers operating on object level.

And I think this is the idea which goes beyond the assumption that relativism is simply arbitrary: every observation has to be made by an observing system, by one and not the other, but if systems are in communication, then something emerges which is not arbitrary anymore but depends on its own history, on its own memory. Luhmann in Rasch and Wolfe [56].

Luhmann's system-theoretical approach introduces an infinite regress of observations of observations.

Cognition deals with an external world that remains unknown and has to, as a result, come to see that it cannot see what it cannot see. Luhmann [55] p. 65.

Which is in concord with Kant's thinking:

(...) the representation in itself does not produce the object insofar as its existence is concerned, for we are not here speaking of causality by means of the will. None the less the representation is a priori determinant of the object if it is to be the case that only through the representation is it possible to know the object. Kant [57] B 124-125.

Yet the external world is less and less unknown as we successively explore it and learn about it. We also learn about the functioning of our cognition and its limitations from biology, brain research, neurosciences, cognitive robotics and similar research which also helps us understand the role of an observer.

\section{What can be Seen Looking "From Within" and Looking "From Without”?}

The ultimate purpose of science is to reconstruct a world by clarifying a modality of motion and structure. It is also possible to express virtual worlds and to hopefully synthesize them via mathematics and computers. The latter makes it possible for the 
former to appear in the world. By this act, scientists try to answer the question 'what is reality?’ Tsuda and Ikegami [58].

Tsuda and Ikegami [58] describe two main human perspectives; the one toward the outside world and the other toward the inner world. The view from without (externalism) is a basis of the conventional scientific view of "exophysics". "The view from within” enables consciousness and selfreferential states, as it is a model capable of accommodating an observer [59]. Those two opposite domains meet at an interface which turns out to be essential for our understanding of the world. Rössler [60] emphasizes relativeness of reality to the state of an observer. He promotes the idea that the internal observer is only allowed interface-type objectivity. For Rössler, the interface or "cut" between "the view from within" and the "view from without" is central, as any observed state is relative to the observer's state at the interface [60].

Individual human experiences, qualia, our unique personal feelings, dependent on contingent influences and attitudes are viewed from within and traditionally expressed in the domain of arts. The basis of arts is the ability of communication from one unique individual (or a group of unique individuals) to the public consisting of other unique individuals. Even if no one can deny uniqueness of each human being, there is obviously a possibility of communication of those unique inner experiences in arts. Although we do not know how it feels for anyone else to sense a sweet rose odor, we have an idea what a poet means by saying "a sweet smell of a rose”, because we share the common physical world of objects which include roses and we as humans have similar sensorimotor apparatus which enables smell. In other words we have strong enough reasons to believe that there is something common to us, all unique individuals, when we experience the sweet rose fragrance or anything else that we have a common language for.

Even though the subjective sensations are personal, they may be studied by scientific methods which connect the objects in the world causing those sensations with bodily reactions which are physical manifestations of those sensations (changes in the brain and nervous system activity, other directly related physical changes). Science as we understand it today does not give a first-person account of qualia - how it feels to be someone else feeling the way that someone else feels. But we might be able with help of simulations and other info-computational methods to understand the mechanisms behind different experiences, feelings and mental processes. We may soon be able to say exactly what is going on in the physical world when a person is feeling angry or happy or hurt and if she is seeing a blue or a red object [61]. Science must be able to identify, describe, understand, and predict that which physically implements qualia, although it will not be from the first person perspective but from the usual scientific third-person. Having a close look from within a physical system such as a view by scanning tunneling microscope is still not a subjective but a neutral third person account.

Although many physical phenomena are critically sensitive to the outside interaction of an observer performing observation or measurement, what finally makes scientific result is not a personal account of an individuated observer, but an intersubjective, neutral, reproducible result. However, in the case of observation-hypersensitive systems (quantum mechanics, chaos), the model of an object should be supplemented by the model of an observer, which we may hope to be able to develop within Info-Computationalist approach to the process of observation. 
The mind seems to be a perfect candidate for modeling within. But in order to understand how the mind works we cannot solely rely on what our minds tell us, even though through consensus on different phenomena of subjective experiences we can conclude that "so it probably looks like from inside a human brain, the way human brain presents it to us”. Nevertheless we must not forget that our brains are not scientific instruments. They have developed through evolution to help us survive in different environments. Many diverse evolutionary optimizations and adaptations have lead to the sort of cognition that we have today. Psychologists show us in how many ways our perception deceives us; from optical illusions to deficiencies in perceiving concurrent events, such as the proverbial gorilla passing unnoticed while people are focusing on counting scores. It is obvious that we cannot only ask for introspective accounts as they are too often unreliable, even when they agree among different individuals.

\section{Two Paradigmatic Projects: The Blue Brain Project and The Biosemiotic Study of Genes, Information and Semiosis. Relevance of Info-computationalist Thinking for Scientific Modeling}

The Blue Brain Project http://bluebrain.epfl.ch studies functions of the brain structural units called the neocortical column (NCC) in the cerebral cortex. Millions of these columns compose the brain. At present stage the Blue Brain Project is attempting to accurately model a single NCC of a young rat brain. The project has succeeded to create an artificial column that responds to electrical impulses in the same way as biological one. The virtual copy of an NCC is built down to the molecular level and simulates the real-world electrical activity of rat brain tissue. The Blue Brain Project team hope eventually to use the same simulation just scaled up, to model the whole rat brain and eventually even the human brain. Currently the Blue Brain runs on IBM's Blue Gene supercomputer. The present computing power is just enough to simulate a single cortical column; while modeling millions of columns is beyond the scope of computational capacity of the Blue Gene. It is speculated that among emergent properties of the brain simulation also consciousness will emerge with rat brain simulation connected in sensorimotor loops with a robotic rat.

The Blue Brain project is in many respects an excellent example of classical constructive science [71] with elements such as reproducibility, third person perspective and meticulously performed reconstruction of biological neurons simulated in a great detail computationally on the Blue Gene supercomputer. The triumph of the Blue Brain project was a spontaneous firing of simulated neurons when given electrical stimuli. The way knowledge is generated is typically info-computational, with simulation representing epistemological laboratory of a kind. The prominent role of computerized visualization is also characteristics of Info-Computationalism as well as a mix of knowledge from many different disciplines-from biology, neuroscience to computer science implemented in info-computational tools, among others in advanced "patch clamp" robot technique contributing to vast effectivization by automatization of experimental procedure combining functions of a microscope with computer-assisted reconstruction of neuron structure.

Nowhere in this research methodology do we see a first person perspective approach. If in the rat simulation consciousness eventually emerges, the simulated rat itself may have "a first person perspective”, but that phenomenon can again be addressed by scientific method of the experiment in which a third person perspective is consequently maintained. 
The next example of a new kind of science based on info-computational understanding is found in Biosemiotics which investigates semiotic processes in living systems, addressing meaning, signification, communication, and habit formation, as well as the physicochemical conditions for sign action and interpretation [62].

Knowledge about biosemiosis, or sign action in living systems combines molecular biology, cognitive ethology, cognitive science, robotics, and neurobiology as they deal with information processes at various levels, which taken together provide a richer foundation for biology. El-Hani, Queiroz and Emmeche [62] go on to describe their approach:

Today, a number of researchers consider information talk as inadequate and 'just metaphorical', expressing a skepticism about the use of the term 'information' and its derivatives in biology as a natural science. We disagree with this position, claiming instead that the notion of information and other related ideas grasp some fundamental features of biological systems and processes that might be otherwise neglected. Our problem is not to get rid of information talk, but rather to clarify it by using a proper theoretical framework. We intend to show that the use of semiotic concepts and theories to interpret information talk can contribute to the construction of a precise and coherent account of information in biology. For this purpose, we introduce here a model of information as semiosis, grounded on Peircean semiotics.

and:

This model is consistent with the best scientific understanding and yet non-reductionist, integrating notions of signs, molecules, and natural interpretation in the tradition of the general semiotics, or the sign theory of Charles Sanders Peirce. It offers a new solution to how to understand and define "gene" in biology, and it develops a profound proposal for another concept of information that does not reduce information to digital bits of computation, but sees it as closely related to natural forms and processes, as the ones known from living organisms.

The above biosemiotic research illustrates how info-computational mechanisms, structures and processes, on different levels of abstraction/description/organization can be seen as constituting biosemiosis by natural computation. In the layered architecture of information, the simplest architectonic elements are data.

What is considered a datum is context-dependent. Once the frame of reference is chosen, potential data can be identified. Given the data (signals or atomic facts), information is obtained by connecting those data with the rest of information/knowledge we have, finding the place for the new pieces of information as meaningful data which make sense in the context of already known. What gives meaning to the data is its relationship with other data, information, knowledge, patterns of behaviors and practices of the community of agents which share the same (or closely related) frame of reference. This is basically what physicist Niels Bohr has found to be valid in physics as well:

Bohr's views can be roughly summarized as follows: Communicable sets of interpreted perceptions are data. Communicable sets of interpreted data are information. Communicable sets of interpreted information are knowledge. Theoretical and contextual 
issues are involved at every step - but different theories are often involved at the different levels. McEvoy [63].

En passant, interesting to note in the above biosemiotic work is that the concepts of information and computation are strongly entangled and sometimes information is characterized as a process while computation is taken to be a structure. Within Info-Computationalism we would talk about computation as semiosis. The information/computation entanglement is understandable knowing the fundamental inseparability of the two, and the fact that biological structural elements at one level of organization serve as programs at the next levels of organization, as already mentioned by von Foerster:

I hope that this simple example is sufficient to suggest to you the possibility of generalizing this principle in the sense that "computation" can be seen on at least two levels, namely, (a) the operations actually performed, and (b) the organization of these operations represented here by the structure of the nerve net. In computer language (a) would again be associated with .operations, but (b) with the .program. As we shall see later, in "biological computers" the programs themselves may be computed on. This leads to the concepts of "meta-programs", "meta-meta-programs", .. . etc. This, of course, is the consequence of the inherent recursive organization of those systems [64].

Similar thoughts may be found in Kampis' [65] component systems models and Sloman and Chrisley’s [66] virtual machines.

\section{Conclusions}

That which Brier identifies as insufficiency of information is significant in several ways. Central are the limits of scientific (third-person) accounts:

Chemical processes and structures can describe and explain crucial aspects of the physiological structure as well as the processes of the nervous system that are necessary for first-person experiences, but chemistry cannot describe the experience as such. Brier [1].

Indeed the essential claim here can be identified as the inability of "knowing from without" (externalist, naturalized, scientific approach) to account for "knowing from within"-subjective, personal experience. This insufficiency may be remedied by taking into account "knowing from within" in at least two ways described as follows.

A) The "first-person account" is included as a "third-person account" of a "first-person account". In the classical view of science, through the mechanism of inter-subjectivity and the requirement of reproducibility of results, specific subjective components in a researcher's experience of the world are washed out from scientific accounts. First order accounts are for obvious reasons important in the fields like medicine, psychology, psychiatry and neuroscience, where they help the construction of a new knowledge about the relationships between the human mind and body. Scientific methods allow for diversity in the context of scientific discovery where among others first order accounts can be applied, while according to Reichenbach in the context of justification there are strict rules of logic and 
inter-subjectivity that will establish if the hypothesis should be accepted or not. The scientist in general is not a part of the story of science. In a big research projects like Blue Brain this is a blessing, as having every researcher's personal account from within his or her total experience of the world would be extremely complex even if handled by the most powerful supercomputers.

Having said that, one must nevertheless admit that there exists strong interest in understanding of the role of an observer (and cognizer) in the process of observation/cognition. With the development of cognitive sciences and cognitive robotics, we are becoming increasingly aware of the fact that we as human beings impose limitations on what may be observed and understood. Up to now sciences have tried to solve the problem of our limited perceptive capabilities by constructing instruments (microscopes, telescopes, infrared cameras, etc.) which help us observe what we otherwise are unable to perceive). On top of that extended perception, there is a need to augment our awareness, reasoning, symbol-manipulation and similar info-computational cognitive capabilities, which will further enhance our repertoire of epistemological tools. Already now we externalize our cognitive functions; we use computers to perform large computations, including diverse simulations and we use networks of connected computing machines to communicate globally. The role of all those new elements in knowledge production which represent mechanisms of extended mind, Clark and Chalmers [67], is still not understood systematically and it is yet to be included in our understanding of science and the epistemic role of researchers as cognitive agents engaged with knowledge production. In sum, by studying our human epistemological characteristics and limitations, along with the strategies of augmented mind [68], we may learn about the possibilities and consequences of expanding our present day cognitive capacities. This will result in a new (second order) scientific knowledge about knowledge generation.

B) A transdisciplinary mixture of different knowledge-production cultures will bring in "knowledge from within" into traditionally externalist fields with new emerging disciplines as a result.

Our idea of knowledge is gradually changing, as we learn about the mechanisms of knowledge production. This process which we already may notice in practice concerns even the evolution of the understanding of science - what science is and how it may develop in the future. Dodig Crnkovic [69] addresses the phenomenon of computing sciences becoming more and more the contemporary ideal of science and thus replacing physics which through the centuries epitomized the ideal of science. Computing sciences/Informatics differ from the classical idea of scientific research in their having human/user/agent in the center of interest. This informational agent is part of the info-computational universe. Inter-disciplinary, cross disciplinary and trans-disciplinary approaches are characteristics of this framework. For Galileo the Great Book of Nature was written in the language of Mathematics. For us today the reality is info-computational and the nature is a computational network best studied by computer simulation combined with direct investigations in the physical world (Fredkin, Chaitin, Wolfram, Lloyd). The information and computation are present in all sciences and other scholar fields, as well as in arts and culture in general. Communication (information exchange) is becoming global and plays an increasingly important role in knowledge production. The boundaries of new knowledge production fields are more fluid and fields mix easily, influencing and reinforcing each other, forming new branches such as cognitive robotics, artificially intelligent environments and simulated virtual worlds. From this development the new insights are expected in the nature of knowledge and its relationships to agency and intentionality. 


\section{Acknowledgements}

The author would like to thank Vincent Müller for numerous enlightening discussions on the foundations of Info-Computationalism, Søren Brier for his helpful and constructive criticism and my 2009 class in Computing and Philosophy with whom I among others repeatedly scrutinized the ideas of second order cybernetics and the epistemological role of the observer. I also wish to thank three anonymous referees for their insightful comments and suggestions.

\section{References and Notes}

1. Brier, S. Cybersemiotics. Why Information Is Not Enough! University of Toronto Press: London, UK, 2008.

2. Qvortrup, L. The Hypercomplex Society; Peter Lang Publishing Co: New York, NY, USA, 2003.

3. Throughout the text, the word "mechanism" is used to stand for not only mechanistic deterministic mechanisms, but any sort of scheme which explains how/why things happen, i.e. the way the concept of "mechanism" is used in physics - as a generator of function and structure. In this sense "Higgs mechanism" refers to spontaneous electroweak symmetry breaking, while “autopoesis” signifies a “mechanism of self-organization”.

4. Chalmers, D. The Conscious Mind: In Search of a Fundamental Theory; Oxford University Press: Oxford, UK, 1996.

5. From the perspective of Artificial Intelligence it would be a great achievement to develop machines that convincingly appear to have general intelligence, even though they would not be able of any first-person accounts. (I strongly doubt the possibility of having full functionality of general intelligence without having ability of a first-person account, but that is a topic for a whole new article). Be that as it may, if a machine appears to learn, we say it learns. It is called machine learning.

6. Anderson, P.W. More is dfferent. Science, 1972, 177, 393-396.

7. Prigogine, I.; Stengers, I. Order Out of Chaos: Man's New Dialogue with Nature; Bantam Books: New York, NY, USA, 1984.

8. Dodig-Crnkovic, G. Investigations into Information Semantics and Ethics of Computing; Mälardalen University Press: Västerås, Sweden, 2006; Available online: http://mdh.divaportal.org/smash/get/diva2:120541/FULLTEXT01 (accessed on 08 March 2010).

9. Dodig-Crnkovic, G.; Müller, V. A Dialogue Concerning Two World Systems: InfoComputational vs. Mechanistic. In Information and Computation; Dodig-Crnkovic G. and Burgin M. Eds.; World Scientific Publishing Co.: Singapore 2010.

10. Floridi, L. A Defence of Informational Structural Realism. Synthese 2008, 161, 219-253.

11. Floridi, L. Against digital ontology. Synthese 2009, 168, 151-178.

12. Chaitin, G. Epistemology as Information Theory, In Computation, Information, Cognition-The Nexus and The Liminal; Dodig-Crnkovic, G., Stuart, S., Eds.; Cambridge Scholars Publishing: Cambridge, UK, 2007; pp. 2-18. Available online: http://www.cs.auckland.ac.nz/ $\sim$ chaitin/ecap.html (accessed on 8 March 2010).

13. Wolfram, S. A New Kind of Science; Wolfram Media: Urbana-Champaign, IL, USA, 2002. 
14. Lloyd, S. Programming the Universe: A Quantum Computer Scientist Takes on the Cosmos; Knopf Publishing Group: New York, NY, USA, 2006.

15. Brier, S. Cybersemiotics and the question of knowledge. In Information and Computation; DodigCrnkovic, G., Burgin, M., Eds.; World Scientific Publishing Co.: Singapore, 2010.

16. Tofoli, T. Nothing makes sense in computing except in the light of evolution. Int. J. Unconvent. Comput. 2005, 1, 1-29.

17. It is important to understand that "efficient cause" does not entail determinism. Aristotle presents his account of the four causes in Physics II 3 and Metaphysics V 2. He recognizes four types of things that can be given in answer to a why-question (Stanford Encyclopedia of Philosophy http://plato.stanford.edu/entries/aristotle-causality/):

- The material cause, that out of which

- The formal cause, the form, the account of what-it-is-to-be, e.g., the shape

- The efficient cause, the primary source of the change or rest and

- The final cause, the end, that for the sake of which a thing is done (or the things happen) Info-Computationalism presents a very suitable framework for understanding of all four causes including the final cause. Living organisms, having memory and intentionality (both eminently info-computational phenomena) are subject to all four causes, downward causation being the results of a living organism's ability to memorize, anticipate and control its own behavior.

18. Objection by Joseph Brenner: "This would exclude all non-Markovian processes in the world which do not depend only on their current state for their evolution." How to answer this objection? We can think in terms of Agent-Based Modeling (ABM) which is modeling phenomena as dynamical systems of interacting agents. Agents have identity and act autonomously. (so e.g., Wolfram's Cellular Automata are agents who act synchronously.) In general one assumes asynchrony and one models environment explicitly. ABM models go beyond state-determined automata by the inclusion of random-access memory capabilities. Agents can engage with their environments beyond concurrent state-determined interaction by using memory to store descriptions and representations of their environments. They can also have access to shared knowledge among the members of their particular agent society. Such agents are dynamically incoherent in the sense that their next state or action is not solely dependent on the previous state but rather depend also on some (random-access) memory that keeps the same value until it is accessed and that does not change with the dynamics of the environment-agent interactions. In the computing universe including the brain, the same hardware that performs computations is used as the memory as well.

19. Dodig-Crnkovic, G. Information and Computation Nets; VDM Verlag: Saarbrücken, Germany, 2009.

20. Knick, M.; Radermacher, F. J. Integration of subsymbolic and symbolic information processing in robot control. In Proceedings of the 3rd Annual Conference on AL, Simulation and Planning in High Autonomy Systems; IEEE Computer Society Press: Washington, USA, 1992; pp 238-243.

21. Results of observations of the same physical object (celestial body) in different wavelengths (radio, microwave, infrared, visible, ultraviolet and X-ray), as in http://bb.nightskylive.net/asterisk/viewtopic.php? $\mathrm{f}=8 \& \mathrm{t}=14561$ illustrates how information in the 
world (multifacetous proto-information) for an observer becomes specific information through the process of making differences by data/signal processing in a given context with a particular observation tools (body and instruments).

22. Dodig-Crnkovic, G. Knowledge generation as natural computation. J. Syst. Cybern. Inform. 2008, 6, 12-16.

23. Burgin, M. Information Dynamics in a Categorical Setting. In Information and Computation; Dodig-Crnkovic, G., Burgin M., Eds.; World Scientific Publishing Co.: Singapore, 2010.

24. MacLennan, B. Natural computation and non-turing models of computation. Theor. Comput. Sci., 2004, 317, 115-145.

25. Zeilinger, A. The message of the quantum. Nature 2005, 438, 743.

26. Emmeche, C. The Garden in the Machine: The Emerging Science of Artificial Life; Princeton University Press: Princeton, NJ, USA, 1994.

27. Gell-Mann, M. The Quark and the Jaguar: Adventures in the Simple and the Complex; Owl Books: New York, NY, USA, 1995.

28. Goertzel, B. The Evolving Mind; Gordon and Breach: New York, NY, USA, 1993.

29. Hoffman, D. The interface theory of perception: natural selection drives true perception to swift extinction. In Object Categorization: Computer and Human Perspectives; Cambridge University Press: Cambridge, UK, 2009.

30. Minsky, M. Interior grounding, reflection, and self-consciousness. In Information and Computation; Dodig-Crnkovic, G., Burgin, M., Eds.; World Scientific Publishing Co.: Singapore, 2010.

31. Wegner, P. Interactive foundations of computing. Theor. Comput. Sci. 1998, 192, 315-351.

32. Kurzweil, R. The Singularity is Near; Viking: New York, NY, USA, 2005.

33. Burgin, M. Super-Recursive Algorithms; Springer Monographs in Computer Science: New York, NY, USA, 2005.

34. Kornblith, H. Knowledge in humans and other animals. Noûs 1999, 33, 327.

35. Harms, W. F. Information and Meaning in Evolutionary Processes; Cambridge University Press: Cambridge, UK, 2004.

36. Harms, W. F. Naturalizing epistemology: prospectus 2006. Biol. Theor. 2006, 1, 23-24.

37. Okasha, S. Review of William F. Harms, Information and meaning in evolutionary processes. Notre Dame Phil. Rev. 12 December 2005.

38. Scripps Research Institute (3 January 2010), 'Lifeless' Prions Capable of Evolutionary Change and Adaptation, Science Daily, Retrieved 12 January 2010; Available online: http://www.sciencedai ly.com/releases/2009/12/091231164747.htm (accessed on 8 March 2010).

39. Bates, M. J. Information and Knowledge: An Evolutionary Framework for Information Science. Inf. Res. 2005, 10; Available online: http://nformationR.net/ir/10-4/paper239.html (accessed on 8 March 2010).

40. Bickhard, M. H. The dynamic emergence of representation. In Representation in Mind: New Approaches to Mental Representation; Clapin, H., Staines, P., Slezak, P., Eds.; Elsevier: Amsterdam, Netherlands, 2004; pp. 71-90. 
41. Kulakov, A.; Stojanov, G. Structures, inner values, hierarchies and stages: essentials for developmental robot architecture, In Proceedings of the Second International Workshop on Epigenetic Robotics: Modeling Cognitive Development in Robotic Systems, Edinburgh, Scotland, 2002; pp. 63-69.

42. Whitehead, A.N. Process and Reality: An Essay in Cosmology; The Free Press: New York, NY, USA, 1978.

43. Flake, G. W. The Computational Beauty of Nature: Computer Explorations of Fractals, Chaos, Complex Systems, and Adaptation; MIT Press: Cambridge, MA, USA, 1998.

44. Gärdenfors, P. How Homo Became Sapiens: On the Evolution of Thinking; Oxford University Press: Oxford, UK, 2003.

45. Stuart, S. The Self as an embedded agent. Mind. Mach. 2003, 13, 187.

46. Maturana, H.; Varela, F. Autopoiesis and Cognition: the Realization of the Living. Cohen, R.; Wartofsky, M.; Eds.; Boston Studies in the Philosophy of Science 42; D. Reidel Publishing: Dordrecht, Netherlands, 1980.

47. Maturana, H.R.; Varela, F.J. The Tree of Knowledge: The Biological Roots of Human Understanding; New Science Library: Boston and London, (Original work published 1984), 1987.

48. Kaneko, K.; Tsuda, I. Complex Systems: Chaos and Beyond. A Constructive Approach with Applications in Life Sciences; Springer: Berlin/Heidelberg, Germany, 2001.

49. Ballard, D. Our perception of the world has to be an illusion. J. Conscious. Stud. 2002, 9, 54-71.

50. Nørretranders, T. The User Illusion: Cutting Consciousness Down to Size; Viking: New York, NY, USA, 1999.

51. Foerster, H. von. Understanding Understanding: Essays on Cybernetics and Cognition; Springer: NewYork, NY, USA, 2003.

52. Heylighen, F.; Joslyn, C. Cybernetics and second order cybernetics. In Encyclopedia of Physical Science \& Technology, 3rd ed.; Meyers, R.A., Ed.; Volume 4, 2001; Academic Press: New York, NY, USA; pp. 155-170. Available online: http://pespmc1.vub.ac.be/Papers/Cybernetics-EPST.pdf (accessed on 8 March 2010).

53. Von Glasersfeld, E. Radical Constructivism: A Way of Knowing and Learning; Falmer Press: London, UK, 1995.

54. Glanville, R. The purpose of second-order cybernetics. Kybernetes 2004, 33, 1379-1386.

55. Luhmann, N. The cognitive program of constructivism and a reality that remains unknown. In Selforganization. Portrait of a Scientific Revolution; Krohn, W., Ed.; Kluwer: Dordrecht, Netherlands, 1990; pp. 64-85.

56. Rasch, W.; Wolfe, C. Observing Complexity: Systems Theory and Postmodernity; University of Minnesota Press: Minneapolis, MN, USA, 2000.

57. Kant, I. Critique of Pure Reason; St Martin's Press: New York, NY, USA, 1965.

58. Tsuda, I.; Ikegami, T. Endophysics: The world as an interface. Discrete Dyn. Nat. Soc., 2002, 7, 213-214.

59. Varela, F.J.; Shear, J. The View from Within: First-person Approaches to the Study of Consciousness; Imprint Academic: Thorverton, UK, 1999.

60. Rössler, O.E. Endophysics, The World as an Interface; World Scientific: Singapore, 1998. 
61. Objection can be made that functional description of the physiology corresponding to qualia is not enough but as long as the connection is established between the event in the world (green light) and the detection of the induced bodily response (seeing green), the observed response is as good as anyone's personal account of the experience of seeing green. In this context measurable events in the world (green light, physiological reaction) are preferable for scientific analysis.

62. El-Hani, C.N.; Queiroz, J.; Emmeche, C. Genes, Information, and semiosis. In Tartu Semiotics Library 8; Tartu University Press: Tartu, Finland, 2009.

63. McEvoy, P. Niels Bohr: Reflections on Subject and Object; MicroAnalytix: New York, NY, USA, 2001.

64. Von Foerster, H. On constructing a reality. In Environmental Design Research; F.E. Preiser (Hg.), Dowden, Hutchinson \& Ross, Stroudberg, 1973; Volume 2, pp. 35-46,

65. Kampis, G. Self-Modifying Systems in Biology and Cognitive Science: A New Framework for Dynamics, Information and Complexity; Pergamon Press: Oxford, UK, 1991.

66. Sloman, A.; Chrisley, R. Virtual Machines and Consciousness, In Journal of Consciousness Studies, 2003, 10, No. 4-5, (Special issue on Machine Consciousness, edited by O. Holland); Available online: http://www.cs.bham.ac.uk/research/projects/cogaff/sloman-chrisley-jcs.pdf (accessed on 8 March 2010).

67. Clark, A.; Chalmers, D. J. The extended mind. Analysis 1998, 58, 10-23.

68. Here Søren Brier reminds me that I never made explicit the position of Info-compuationalism in question of the relationship between mind and brain. As it is possible to reconstruct from fragments of naturalized epistemology, the position is basically one of eliminativism, close to the views of Dennett, Hofstadter and the Churchlands - mental states are identical with brain states in a sense of Sloman's virtual machine.

69. Dodig-Crnkovic, G. Shifting the Paradigm of the Philosophy of Science: the Philosophy of Information and a New Renaissance, Mind. Mach., 2003, 13, Issue 4; Available online: http://www.springerlink.com/content/g14t483510156726 (accessed on 8 March 2010).

70. Hofkirchner, W. Does computing embrace self-organisation? In Information and Computation; Dodig-Crnkovic, G., Burgin, M., Eds.; World Scientific Publishing Co.: Singapore, 2010.

71. Dodig-Crnkovic, G. Constructive research and info-computational knowledge generation. In Proceedings Model-Based Reasoning in Science and Technology. Abduction, Logic, and Computational Discovery Conference 2009, Campinas, Brazil; Springer series "Studies in Computational Intelligence" forthcoming 2010.

(C) 2010 by the authors; licensee Molecular Diversity Preservation International, Basel, Switzerland. This article is an open-access article distributed under the terms and conditions of the Creative Commons Attribution license (http://creativecommons.org/licenses/by/3.0/ 